\title{
Vacuum polarization of a scalar field in wormhole spacetimes
}

\author{
Arkadii A. Popov*, Sergey V. Sushkov ${ }^{\dagger}$ \\ Department of Geometry, Kazan State Pedagogical University, \\ Mezhlauk 1 st., Kazan 420021, Russia
}

\begin{abstract}
An analitical approximation of $\left\langle\phi^{2}\right\rangle$ for a scalar field in a static spherically symmetric wormhole spacetime is obtained. The scalar field is assumed to be both massive and massless, with an arbitrary coupling $\xi$ to the scalar curvature, and in a zero temperature vacuum state.
\end{abstract}

PACS number(s): 04.62.+v, 04.70.Dy

\section{INTRODUCTION}

Wormholes are topological handles in spacetime linking widely separated regions of a single universe, or "bridges" joining two different spacetimes [1]. Interest in these configurations dates back at least as far as 1916 [2] with punctuated revivals of activity following both the classic work of Einstein and Rosen in 1935 [3] and the later series of works initiated by Wheeler in 1955 迎. More recently, a fresh interest in the topic has been rekindled by the works of Morris and Thorne [5] and of Morris, Thorne and Yurtsever [6]. These authors constructed and investigated a class of objects they referred to as "traversable wormholes." Their work led to a flurry of activity in wormhole physics [7].

One of the most central feature of wormhole physics is the fact that traversable wormholes are accompanied by unavoidable violations of the null energy condition (NEC), i.e., the matter threading the wormhole's throat has to be possessed of "exotic" properties. The known classical matter does satisfy the usual energy conditions, hence wormholes, if they exist, should arise as solutions of general relativity and "unusal" and/or quantum matter.". Recently, Barceló and Visser 13,14 have demonstrated the possibility of existence of traversable wormholes supported by classical nonminimally coupled scalar fields. In their investigation, scalar fields play a role of the "unusual" matter violating the NEC, and therefore, instead of the problem of the wormhole's existence, the problem of the existence of appropriate scalars comes to the forefront.

An alternative approach is to regard wormholes as semiclassical in nature, and seek them as solutions of the Einstein equations, using the expectation value of the stress-energy tensor as the source of gravity (self-consistent wormholes). Such an idea first appeared and was realized in Ref. [15]. Note that the main difficulty of this approach is that the expectation values have the strong functional dependence on the metric tensor $g_{\mu \nu}$ and are generally impossible to be calculated analitically. For this reason, one is forced to use approximate expressions for the expectation value of the stress-energy tensor. In Ref. [15], the Frolov-Zel'nikov approximation [16] for $\left\langle T_{\mu \nu}\right\rangle$ for conformally invariant massless fields in static spacetimes, based on pure geometrical arguments and common properties of the stress-energy tensor, has been used. Later, in Ref. [17] self-consistent spherically symmetric wormhole solutions have been found numerically in the framework of the analytical approximation for $\left\langle T_{\mu \nu}\right\rangle$, which was derived by Anderson, Hiscock and Samuel [18] on the basis of the WKB approximation for modes of a scalar field. Recently Taylor, Hiscock, and Anderson [19], using the Dewitt-Schwinger approximation, have calculated analytic expressions for the stressenergy of a quantized massive scalar field in a number of static spherically symmetric wormhole spacetimes. As well some arguments in favour of the possibility of existence of self-consistent wormhole solutions to semiclassical Einstein equations have been given in Ref. [2]].

Recently one of the authors [21] has performed approximate calculations of $\left\langle\phi^{2}\right\rangle$ for a massive scalar field in static spherically symmetric spacetimes, which improve the result by Anderson, Hiscock, and Samuel [18]. The improvement has been achieved due to the use of exact expressions for mode sums and integrals. Moreover, it has been demonstrated in Ref. [21] that the result of approximate calculations depends on the appropriate choice of the zeroth-order solution of the mode equation. In this paper we apply the experience obtained in the previous work to derive an approximate expression for $\left\langle\phi^{2}\right\rangle$ for a scalar field in a wormhole spacetime.

\footnotetext{
*e-mail: popov@kspu.kcn.ru

${ }^{\dagger}$ e-mail: sushkov@kspu.kcn.ru

${ }^{1}$ The NEC can also be violated and wormholes can exist in theories based on modifications of general relativity, such as Brans-Dicke theory [8 10], higher derivative gravity [1] or Gauss-Bonnet theory [12].
} 


\section{WORMHOLE SPACETIMES}

We shall regard a wormhole as a time independent, nonrotating, and spherically symmetric bridge connecting two asymptotically flat regions. The metric of wormhole spacetime (continued analitically into Euclidean space) can be taken in the form, suggested by Morris and Thorne [5]:2]

$$
d s^{2}=f(\rho) d \tau^{2}+d \rho^{2}+r^{2}(\rho)\left(d \theta^{2}+\sin ^{2} \theta d \varphi^{2}\right)
$$

Here $\tau=i t$ is the Euclidean time, $\rho$ is the proper radial distance, $\rho \in(-\infty,+\infty)$. We assume that the redshift function $f(\rho)$ is everywhere finite (no event horizons); the shape function $r(\rho)$ has the global minimum at $\rho=0$, so that $r_{0}=\min \{r(\rho)\}=r(0)$ is the radius of the wormhole throat. The topology of the wormhole spacetime is $R^{2} \times S^{2}$. In order for the spacetime geometry to tend to an appropriate asymptotically flat limit at $\rho \rightarrow \pm \infty$ we impose

$$
\lim _{\rho \rightarrow \pm \infty}\{r(\rho) /|\rho|\}=1, \quad \text { and } \quad \lim _{\rho \rightarrow \pm \infty} f(\rho)=f_{ \pm} .
$$

For simplicity we also assume symmetry under interchange of the two asymptotically flat regions, $\rho \leftrightarrow-\rho$, that is, $r(\rho)=r(-\rho)$ and $f(\rho)=f(-\rho)$.

Note that introducing a new radial variable $r$ instead of $\rho$ by substitution

$$
r=r(\rho)
$$

allows to rewrite the metric (11) in the following form

$$
d s^{2}=f(r) d \tau^{2}+h(r) d r^{2}+r^{2}\left(d \theta^{2}+\sin ^{2} \theta d \varphi^{2}\right)
$$

with

$$
h(r)=\frac{1}{r^{\prime 2}}
$$

where the prime means the derivative with respect to $\rho$.

\section{APPROXIMATE EXPRESSION FOR $\left\langle\phi^{2}\right\rangle$}

Consider a quantized scalar field $\phi$; the scalar field is assumed to be both massive with the mass $m$ and massless, and with an arbitrary coupling $\xi$ to the scalar curvature. We also assume that the field is in a vacuum state defined with respect to the Killing vector which always exists in a static spacetime. An unrenormalized expression for $\left\langle\phi^{2}\right\rangle$ can be computed from the Euclidean Green's function $G_{E}(x, \tilde{x})$. Such the expression given in Ref. [18] for the case of static spherically symmetric spacetimes reads

$$
\begin{aligned}
\left\langle\phi^{2}(x)\right\rangle_{\text {unren }} & =G_{E}(x, \tau ; x, \tilde{\tau}) \\
& =\frac{1}{4 \pi^{2}} \int_{0}^{\infty} d \omega \cos [\omega(\tau-\tilde{\tau})] \sum_{l=0}^{\infty}\left[(2 l+1) p_{\omega l} q_{\omega l}-\frac{1}{r f^{1 / 2}}\right]
\end{aligned}
$$

where the modes $p_{\omega l}$ and $q_{\omega l}$ obey the homogeneous radial mode equation

$$
\frac{d^{2} S}{d \rho^{2}}+\left[\frac{1}{2 f} \frac{d f}{d \rho}+\frac{2}{r} \frac{d r}{d \rho}\right] \frac{d S}{d \rho}-\left[\frac{\omega^{2}}{f}+\frac{l(l+1)}{r^{2}}+m^{2}+\xi R\right] S=0
$$

The modes $p_{\omega l}$ and $q_{\omega l}$ also satisfy the Wronskian condition

$$
C_{\omega l}\left[p_{\omega l} \frac{d q_{\omega l}}{d \rho}-q_{\omega l} \frac{d p_{\omega l}}{d \rho}\right]=-\frac{1}{r^{2} f^{1 / 2}}
$$

\footnotetext{
${ }^{2}$ The units $\hbar=c=G=1$ are used throughout the paper.
} 
where $C_{\omega l}$ is a normalization constant.

Let us stress that points in Eq. (19) are splitted. Namely, points are separated in time so that $\epsilon \equiv(\tau-\tilde{\tau}), \tilde{\rho}=\rho$, $\tilde{\theta}=\theta, \tilde{\varphi}=\varphi$. As was first pointed out by Candelas and Howard [25] for the case of Schwarzschild spacetime, the Euclidean Green's function have superficial divergences with this separation of points. As discussed in Ref. [18], these cannot be real divergences because the Green's function must be finite when the points are separated; to remove the divergences one has to subtract some additional counterterms. The terms $\left(r f^{1 / 2}\right)^{-1}$ in brackets in Eq. (19) are such counterterms (for details, see discussion in Ref. [18]).

Now let us proceed to the WKB representation by making the change of variables

$$
\begin{aligned}
& p_{\omega l}=\frac{1}{\left(2 r^{2} W\right)^{1 / 2}} \exp \left[\int^{\rho} W f^{-1 / 2} d \rho\right], \\
& q_{\omega l}=\frac{1}{\left(2 r^{2} W\right)^{1 / 2}} \exp \left\{-\left[\int^{\rho} W f^{-1 / 2} d \rho\right]\right\},
\end{aligned}
$$

where $W$ is a new function of $\rho$. Substitution of Eq. (8) into Eq. (7) shows that the Wronskian condition is obeyed if $C_{\omega l}=1$, and substitution of Eq. (8) into Eq. (6) gives the following equation for $W$ :

$$
W^{2}=\omega^{2}+\frac{f}{r^{2}} l(l+1)+m^{2} f+\xi f R+\frac{r^{\prime} f^{\prime}}{2 r}+\frac{r^{\prime \prime} f}{2 r}+\frac{f^{\prime}}{4} \frac{W^{\prime}}{W}+\frac{f}{2} \frac{W^{\prime \prime}}{W}-\frac{3 f}{4} \frac{W^{\prime 2}}{W^{2}}
$$

with

$$
R=-\frac{f^{\prime \prime}}{f}+\frac{f^{\prime 2}}{2 f^{2}}-\frac{2 r^{\prime} f^{\prime}}{r f}-\frac{4 r^{\prime \prime}}{r}-\frac{2 r^{\prime 2}}{r^{2}}+\frac{2}{r^{2}}
$$

Assuming that the functions $f$ and $r$ are varying sufficiently slowly, one can solve Eq. (9) iteratively with the small parameter

$$
\lambda_{W K B}=L_{\star} / L \ll 1,
$$

where $L$ is a characteristic scale of varying of the metric functions 3 and

$$
L_{\star}=\left[m^{2}+\frac{2 \xi}{r^{2}}\right]^{-1 / 2}
$$

Neglecting terms with derivatives in Eq. (9) we choose the zeroth-order solution as follows:

$$
W=\Omega
$$

where

$$
\begin{aligned}
\Omega & =\left[\omega^{2}+\frac{f}{r^{2}} l(l+1)+f m^{2}+\frac{2 \xi}{r^{2}}\right]^{1 / 2} \\
& =\left[\omega^{2}+\frac{f}{r^{2}}\left(l+\frac{1}{2}\right)^{2}+\frac{f}{r^{2}} \mu^{2}\right]^{1 / 2} .
\end{aligned}
$$

Here we denote

$$
\mu^{2}=m^{2} r^{2}+2 \delta
$$

with $\delta=\xi-1 / 8$. Stress that below we assume $\mu^{2}>0$. The fourth-order solution is

$$
W=\Omega+W^{(2)}+W^{(4)}
$$

\footnotetext{
${ }^{3}$ More exactly, one may define $L$ as follows: $L^{-1}=\max \left\{\left|[\ln (f r)]^{\prime}\right|,\left|[\ln (f r)]^{\prime \prime}\right|^{1 / 2},\left|[\ln (f r)]^{\prime \prime \prime}\right|^{1 / 3}, \ldots\right\}$.
} 
with

$$
W^{(2)}=\frac{1}{2} \frac{V}{\Omega}+\frac{f}{4} \frac{\Omega^{\prime \prime}}{\Omega^{2}}+\frac{f^{\prime}}{8} \frac{\Omega^{\prime}}{\Omega^{2}}-\frac{3 f}{8} \frac{\Omega^{\prime 2}}{\Omega^{3}}
$$

and

$$
W^{(4)}=-\frac{W^{(2)^{2}}}{2 \Omega}+\frac{f}{4} \frac{W^{(2)^{\prime \prime}}}{\Omega^{2}}+\frac{f^{\prime}}{8} \frac{W^{(2)^{\prime}}}{\Omega^{2}}-\frac{f}{4} \frac{\Omega^{\prime \prime} W^{(2)}}{\Omega^{3}}-\frac{f^{\prime}}{8} \frac{\Omega^{\prime} W^{(2)}}{\Omega^{3}}+\frac{3 f}{4} \frac{{\Omega^{\prime}}^{2} W^{(2)}}{\Omega^{4}},
$$

where

$$
V=\frac{r^{\prime} f^{\prime}}{2 r}+\frac{r^{\prime \prime} f}{2 r}+\xi f\left(R-\frac{2}{r^{2}}\right) .
$$

Substituting the solution (15) into (8) and (19), and neglecting terms of the sixth order and higher we can obtain the following expression for the fourth-order WKB approximation for $\left\langle\phi^{2}\right\rangle$ :

$$
\begin{aligned}
\left\langle\phi^{2}\right\rangle_{\text {unren }}= & \frac{1}{4 \pi^{2} r^{2}} \int_{0}^{\infty} d \omega \cos [\omega(\tau-\tilde{\tau})] \sum_{l=0}^{\infty}\left(l+\frac{1}{2}\right)\left\{\left[\frac{1}{\Omega}-\frac{r}{f^{1 / 2}\left(l+\frac{1}{2}\right)}\right]-\frac{V}{2 \Omega^{3}}\right. \\
& +\frac{f^{2}}{\Omega^{5}}\left[-\frac{U^{\prime \prime}}{8 f}-\frac{f^{\prime} U^{\prime}}{16 f^{2}}+\frac{3 V^{2}}{8 f^{2}}-\frac{f^{\prime} V^{\prime}}{16 f^{2}}-\frac{V^{\prime \prime}}{8 f}\right]+\frac{f^{2}}{\Omega^{7}}\left[\frac{5 U^{\prime 2}}{32 f}-\frac{f^{\prime \prime} U^{\prime \prime}}{16 f}-\frac{3 f^{\prime 2} U^{\prime \prime}}{128 f^{2}}\right. \\
& \left.+\frac{5 V U^{\prime \prime}}{16 f}-\frac{f^{\prime \prime \prime} U^{\prime}}{64 f}-\frac{f^{\prime} f^{\prime \prime} U^{\prime}}{128 f^{2}}+\frac{5 V^{\prime} U^{\prime}}{16 f}+\frac{5 f^{\prime} V U^{\prime}}{32 f}-\frac{3 f^{\prime} U^{\prime \prime \prime}}{32 f}-\frac{U^{\prime \prime \prime \prime}}{32}\right] \\
& +\frac{f^{2}}{\Omega^{9}}\left[\frac{7 f^{\prime \prime} U^{\prime 2}}{64 f}+\frac{21 f^{\prime 2} U^{\prime 2}}{512 f^{2}}-\frac{35 V U^{\prime 2}}{64 f}+\frac{63 f^{\prime} U^{\prime} U^{\prime \prime}}{128 f}+\frac{7 U^{\prime} U^{\prime \prime \prime}}{32}+\frac{21 U^{\prime \prime 2}}{128}\right] \\
& \left.+\frac{f^{2}}{\Omega^{11}}\left[-\frac{231 f^{\prime} U^{\prime 3}}{512 f}-\frac{231 U^{\prime 2} U^{\prime \prime}}{256}\right]+\frac{f^{2}}{\Omega^{13}} \frac{1155 U^{\prime 4}}{2048}\right\},
\end{aligned}
$$

where

$$
U=\frac{f}{r^{2}}\left(l+\frac{1}{2}\right)^{2}+f\left(m^{2}+\frac{2 \delta}{r^{2}}\right) .
$$

A renormalized expression for $\left\langle\phi^{2}\right\rangle$ can be obtained as follows:

$$
\left\langle\phi^{2}\right\rangle_{\mathrm{ren}}=\lim _{\epsilon \rightarrow 0}\left(\left\langle\phi^{2}\right\rangle_{\mathrm{unren}}-\left\langle\phi^{2}\right\rangle_{\mathrm{DS}}\right),
$$

where the renormalization counterterms for a massive scalar field are given by [26]

$$
\begin{aligned}
\left\langle\phi^{2}\right\rangle_{\mathrm{DS}} & =G_{\mathrm{DS}}\left(x, x^{\prime}\right) \\
& =\frac{1}{8 \pi^{2} \sigma}+\frac{1}{8 \pi^{2}}\left[m^{2}+\left(\xi-\frac{1}{6}\right) R\right]\left[C+\frac{1}{2} \ln \left(\frac{m_{\mathrm{DS}}^{2}|\sigma|}{2}\right)\right]-\frac{m^{2}}{16 \pi^{2}}+\frac{1}{96 \pi^{2}} R_{\alpha \beta} \frac{\sigma^{\alpha} \sigma^{\beta}}{\sigma} .
\end{aligned}
$$

Here $\sigma$ is equal to one half the square of the distance between the points $x$ and $x^{\prime}$ along the shortest geodesic connecting them. $C$ is Euler's constant, $R_{\alpha \beta}$ is the Ricci tensor and $\sigma^{\alpha} \equiv \sigma^{; \alpha}$. The constant $m_{\mathrm{DS}}$ is equal to the mass $m$ of the field for a massive scalar field. For a massless scalar field it is an arbitrary parameter. A particular choice of the value of $m_{\mathrm{DS}}^{2}$ corresponds to a finite renormalization of the coefficients of terms in the gravitational Lagrangian.

To perform the procedure of renormalization in practice we make use of the formulas obtained in Ref. [21]. These formulas give the expressions for mode sums and integrals as exact expansions in powers of $\epsilon$ :

$$
\begin{aligned}
S_{0}(\epsilon, \mu) & =\int_{0}^{\infty} d u \cos \left(u \frac{\epsilon f^{1 / 2}}{r}\right) \sum_{l=0}^{\infty}\left[\frac{l+1 / 2}{\sqrt{u^{2}+\mu^{2}+(l+1 / 2)^{2}}}-1\right] \\
& =\frac{r^{2}}{f \epsilon^{2}}+\frac{1}{2}\left(\mu^{2}-\frac{1}{12}\right)\left(\ln \frac{\epsilon \mu f^{1 / 2}}{2 r}+C\right)-\frac{\mu^{2}}{4}-\mu^{2} S_{0}(2 \pi \mu)+\mathrm{O}\left(\epsilon^{2} \ln \epsilon\right),
\end{aligned}
$$




$$
\begin{aligned}
& \begin{aligned}
S_{n}(\epsilon, \mu) & =\int_{0}^{\infty} d u \cos \left(u \frac{\epsilon f^{1 / 2}}{r}\right) \sum_{l=0}^{\infty} \frac{(l+1 / 2)^{2 n-1}}{\left[u^{2}+\mu^{2}+(l+1 / 2)^{2}\right]^{n+1 / 2}}, \\
& =-\frac{2^{n-1}(n-1) !}{(2 n-1) ! !}\left(\ln \frac{\epsilon \mu f^{1 / 2}}{2 r}+C\right)+\frac{S_{n}(2 \pi \mu)}{(2 n-1) ! !}+\mathrm{O}\left(\epsilon^{2} \ln \epsilon\right),
\end{aligned} \\
& n=1,2,3, \ldots,
\end{aligned}
$$

where $(2 n-1) ! ! \equiv 1 \cdot 3 \cdot 5 \cdots(2 n-1)$ and

$$
\begin{aligned}
& S_{0}(2 \pi \mu)=\int_{0}^{\infty} \frac{x \ln \left|1-x^{2}\right|}{e^{2 \pi \mu x}+1} d x \\
& S_{n}(2 \pi \mu)=\int_{0}^{\infty} d x \ln \left|1-x^{2}\right| \frac{d}{d x}\left(\frac{1}{x} \frac{d}{d x}\right)^{n-1} \frac{x^{2(n-1)}}{e^{2 \pi \mu x}+1} .
\end{aligned}
$$

Together with the integrosums (23), which diverge in the limit $\epsilon \rightarrow 0$, the expression (19) contains the integrosums of the following form:

$$
\begin{aligned}
& N_{n}^{m}(\epsilon, \mu)=\int_{0}^{\infty} d u \cos \left(u \frac{\epsilon f^{1 / 2}}{r}\right) \sum_{l=0}^{\infty} \frac{(l+1 / 2)^{m}}{\left[u^{2}+\mu^{2}+(l+1 / 2)^{2}\right]^{n+1 / 2}}, \\
& n=2,3,4, \ldots, \\
& m=1,3, \ldots, 2 n-3
\end{aligned}
$$

The functions $N_{n}^{m}(\epsilon, \mu)$ are regular in the limit $\epsilon \rightarrow 0$, and so one may directly set $\epsilon=0$ in Eq. (25). Then, changing the order of summation and integration in Eq. (25) and integrating over $u$ gives

$$
N_{n}^{m}(0, \mu)=\frac{(2 n-2) ! !}{(2 n-1) ! !} N_{n}^{m}(\mu)
$$

with

$$
N_{n}^{m}(\mu)=\sum_{l=0}^{\infty} \frac{\left(l+\frac{1}{2}\right)^{m}}{\left[\mu^{2}+\left(l+\frac{1}{2}\right)^{2}\right]^{n}} .
$$

Substituting Eqs. (23,26) into Eq. (19) we may find the asymptotical expansion for $\left\langle\phi^{2}\right\rangle_{\text {unren }}$ in the limit $\epsilon \rightarrow 0$. Finally, carring out the procedure of renormalization (21), i.e. subtracting $\left\langle\phi^{2}\right\rangle_{\mathrm{DS}}$, we obtain the renormalized expression for $\left\langle\phi^{2}\right\rangle$ in the framework of the fourth-order WKB approximation:

$$
\begin{aligned}
& 4 \pi^{2}\left\langle\phi^{2}\right\rangle_{\text {ren }}=\frac{1}{4}\left[m^{2}+\left(\xi-\frac{1}{6}\right) R\right] \ln \left(\frac{\mu^{2}}{m_{\mathrm{DS}}^{2} r^{2}}\right)-\frac{\delta}{2 r^{2}}-\frac{\mu^{2}}{r^{2}} S_{0}(2 \pi \mu)+\frac{f^{\prime \prime}}{24 f}-\frac{f^{\prime 2}}{24 f^{2}}+\frac{f^{\prime} r^{\prime}}{12 f r} \\
& -\frac{V}{2 f} S_{1}(2 \pi \mu)-S_{2}(2 \pi \mu) \frac{r^{2}}{24 f^{2}}\left[f\left(\frac{f}{r^{2}}\right)^{\prime \prime}+\frac{1}{2} f^{\prime}\left(\frac{f}{r^{2}}\right)^{\prime}\right]+S_{3}(2 \pi \mu) \frac{r^{4}}{96 f^{2}}\left(\frac{f}{r^{2}}\right)^{\prime 2} \\
& -N_{2}^{1}(\mu) \frac{r^{2}}{12 f}\left\{\left(\frac{f}{r^{2}} \mu^{2}\right)^{\prime \prime}+\frac{1}{2} \frac{f^{\prime}}{f}\left(\frac{f}{r^{2}} \mu^{2}\right)^{\prime}-\frac{3 V^{2}}{f}+V^{\prime \prime}+\frac{1}{2} \frac{f^{\prime}}{f} V^{\prime}\right\} \\
& +N_{3}^{1}(\mu) \frac{r^{4}}{12 f^{2}}\left\{\left(\frac{f \mu^{2}}{r^{2}}\right)^{\prime 2}-\left(\frac{\mu^{2} f}{r^{2}}\right)^{\prime}\left(\frac{1}{10} f^{\prime \prime \prime}+\frac{1}{20} \frac{f^{\prime} f^{\prime \prime}}{f}-2 V^{\prime}-\frac{f^{\prime} V}{f}\right)\right. \\
& \left.-\left(\frac{\mu^{2} f}{r^{2}}\right)^{\prime \prime}\left(\frac{2}{5} f^{\prime \prime}+\frac{3}{20} \frac{f^{\prime 2}}{f}-2 V\right)-\frac{3}{5} \frac{f^{\prime}}{f}\left(\frac{\mu^{2} f}{r^{2}}\right)^{\prime \prime \prime}-\frac{1}{5}\left(\frac{\mu^{2} f}{r^{2}}\right)^{\prime \prime \prime \prime \prime}\right\} \\
& +N_{3}^{3}(\mu) \frac{r^{4}}{12 f^{2}}\left\{2\left(\frac{f}{r^{2}}\right)^{\prime}\left(\frac{\mu^{2} f}{r^{2}}\right)^{\prime}-\left(\frac{f}{r^{2}}\right)^{\prime}\left(\frac{1}{10} f^{\prime \prime \prime}+\frac{1}{20} \frac{f^{\prime} f^{\prime \prime}}{f}-2 V^{\prime}-\frac{f^{\prime} V}{f}\right)\right. \\
& \left.-\left(\frac{f}{r^{2}}\right)^{\prime \prime}\left(\frac{2}{5} f^{\prime \prime}+\frac{3}{20} \frac{f^{\prime 2}}{f}-2 V\right)-\frac{3}{5} \frac{f^{\prime} f^{\prime \prime \prime}}{f}-\frac{1}{5} f^{\prime \prime \prime \prime \prime}\right\}
\end{aligned}
$$




$$
\begin{aligned}
& +N_{4}^{5}(\mu) \frac{r^{6}}{10 f^{3}}\left\{\left(\frac{f}{r^{2}}\right)^{\prime 2}\left(\frac{1}{2} f^{\prime \prime}+\frac{3}{16} \frac{f^{\prime 2}}{f}-\frac{5}{2} V\right)+\frac{9}{4} f^{\prime}\left(\frac{f}{r^{2}}\right)^{\prime}\left(\frac{f}{r^{2}}\right)^{\prime \prime}\right. \\
& \left.+f\left(\frac{f}{r^{2}}\right)^{\prime}\left(\frac{f}{r^{2}}\right)^{\prime \prime \prime}+\frac{15}{28} f\left(\frac{f}{r^{2}}\right)^{\prime \prime 2}\right\} \\
& +N_{4}^{3}(\mu) \frac{r^{6}}{10 f^{3}}\left\{\left(\frac{f}{r^{2}}\right)^{\prime}\left(\frac{\mu^{2} f}{r^{2}}\right)^{\prime}\left(f^{\prime \prime}+\frac{3}{8} \frac{f^{\prime 2}}{f}-5 V\right)+\frac{9}{4} f^{\prime}\left(\frac{f}{r^{2}}\right)^{\prime}\left(\frac{\mu^{2} f}{r^{2}}\right)^{\prime \prime}\right. \\
& \left.+\frac{9}{4} f^{\prime}\left(\frac{f}{r^{2}}\right)^{\prime \prime}\left(\frac{\mu^{2} f}{r^{2}}\right)^{\prime}+f\left(\frac{f}{r^{2}}\right)^{\prime}\left(\frac{\mu^{2} f}{r^{2}}\right)^{\prime \prime \prime}+f\left(\frac{f}{r^{2}}\right)^{\prime \prime \prime}\left(\frac{\mu^{2} f}{r^{2}}\right)^{\prime}+\frac{15}{14} f\left(\frac{f}{r^{2}}\right)^{\prime \prime}\left(\frac{\mu^{2} f}{r^{2}}\right)^{\prime \prime}\right\} \\
& +N_{4}^{1}(\mu) \frac{r^{6}}{10 f^{3}}\left\{\left(\frac{\mu^{2} f}{r^{2}}\right)^{\prime 2}\left(\frac{1}{2} f^{\prime \prime}+\frac{3}{16} \frac{f^{\prime 2}}{f}-\frac{5}{2} V\right)+\frac{9}{4} f^{\prime}\left(\frac{\mu^{2} f}{r^{2}}\right)^{\prime}\left(\frac{\mu^{2} f}{r^{2}}\right)^{\prime \prime}\right. \\
& \left.+f\left(\frac{\mu^{2} f}{r^{2}}\right)^{\prime}\left(\frac{\mu^{2} f}{r^{2}}\right)^{\prime \prime \prime}+\frac{3}{4} f\left(\frac{\mu^{2} f}{r^{2}}\right)^{\prime \prime 2}\right\}-N_{5}^{7}(\mu) \frac{11 r^{8}}{30 f^{4}}\left\{\frac{1}{2} f^{\prime}\left(\frac{f}{r^{2}}\right)^{\prime 3}+f\left(\frac{f}{r^{2}}\right)^{\prime 2}\left(\frac{f}{r^{2}}\right)^{\prime \prime}\right\} \\
& -N_{5}^{5}(\mu) \frac{11 r^{8}}{30 f^{4}}\left\{\frac{3}{2} f^{\prime}\left(\frac{f}{r^{2}}\right)^{\prime 2}\left(\frac{\mu^{2} f}{r^{2}}\right)^{\prime}+2 f\left(\frac{f}{r^{2}}\right)^{\prime}\left(\frac{f}{r^{2}}\right)^{\prime \prime}\left(\frac{\mu^{2} f}{r^{2}}\right)^{\prime}+f\left(\frac{f}{r^{2}}\right)^{\prime 2}\left(\frac{\mu^{2} f}{r^{2}}\right)^{\prime \prime}\right\} \\
& -N_{5}^{3}(\mu) \frac{11 r^{8}}{30 f^{4}}\left\{\frac{3}{2} f^{\prime}\left(\frac{f}{r^{2}}\right)^{\prime}\left(\frac{\mu^{2} f}{r^{2}}\right)^{\prime 2}+f\left(\frac{f}{r^{2}}\right)^{\prime \prime}\left(\frac{\mu^{2} f}{r^{2}}\right)^{\prime 2}+2 f\left(\frac{f}{r^{2}}\right)^{\prime}\left(\frac{\mu^{2} f}{r^{2}}\right)^{\prime}\left(\frac{\mu^{2} f}{r^{2}}\right)^{\prime \prime}\right\} \\
& +N_{5}^{1}(\mu) \frac{11 r^{8}}{30 f^{4}}\left\{\frac{1}{2} f^{\prime}\left(\frac{\mu^{2} f}{r^{2}}\right)^{\prime 3}+f\left(\frac{\mu^{2} f}{r^{2}}\right)^{\prime 2}\left(\frac{\mu^{2} f}{r^{2}}\right)^{\prime \prime}\right\} \\
& +N_{6}^{9}(\mu) \frac{5 r^{10}}{24 f^{4}}\left(\frac{f}{r^{2}}\right)^{\prime 4}+N_{6}^{7}(\mu) \frac{5 r^{10}}{6 f^{4}}\left(\frac{f}{r^{2}}\right)^{\prime 3}\left(\frac{\mu^{2} f}{r^{2}}\right)^{\prime}+N_{6}^{5}(\mu) \frac{5 r^{10}}{4 f^{4}}\left(\frac{f}{r^{2}}\right)^{\prime 2}\left(\frac{\mu^{2} f}{r^{2}}\right)^{\prime 2}\left(\frac{\mu^{2} f}{r^{2}}\right)^{\prime 3}+N_{6}^{1}(\mu) \frac{5 r^{10}}{24 f^{4}}\left(\frac{\mu^{2} f}{r^{2}}\right)^{\prime 4} \cdot\left(N^{\prime}\right.
\end{aligned}
$$

\section{ANALYSIS}

The analytical approximation for $\left\langle\phi^{2}\right\rangle$, given by Eq. (27), may be separately analysed for two different cases: (i) $r(\rho) \gtrsim L$, and (ii) $r(\rho) \ll L$.

\section{A. The case $r \gtrsim L$}

First, let us consider $\left\langle\phi^{2}\right\rangle$ in the region $r(\rho) \gtrsim L$. Note that in this case $\mu^{2} \approx m^{2} r^{2} \gg 1$. Really, it immediately follows from the basic WKB condition $\lambda_{W K B}=L_{\star} / L \ll 1$ :

$$
1 \ll \frac{L^{2}}{L_{\star}^{2}}=L^{2}\left(m^{2}+\frac{2 \xi}{r^{2}}\right) \lesssim r^{2}\left(m^{2}+\frac{2 \xi}{r^{2}}\right)=m^{2} r^{2}+2 \xi,
$$

assuming that $\xi$ is of order unity or less, we conclude that $m^{2} r^{2} \gg 1$. The functions $S_{n}(2 \pi \mu)$ and $N_{n}^{m}(\mu)$ have a simple asymptotical form at large values of $m r$; the respective formulas are given in Appendix (see Eqs. (A2), (A7)). Substituting these formulas into Eq. (27) and taking into account the asymptotical expansion $\ln (1+x) \approx x-\frac{1}{2} x^{2}$ gives

$$
\begin{aligned}
& 4 \pi^{2} m^{2}\left\langle\phi^{2}\right\rangle_{\text {ren }}=\frac{5 \xi-1}{120} \frac{f^{\prime \prime \prime \prime}}{f}-\frac{5 \xi-1}{48} \frac{f^{\prime \prime \prime} f^{\prime}}{f^{2}}+\left(-\frac{1}{8} \xi^{2}+\frac{7}{24} \xi-\frac{13}{240}\right) \frac{f^{\prime \prime} f^{\prime 2}}{f^{3}} \\
& +\left(\frac{1}{8} \xi^{2}-\frac{1}{8} \xi+\frac{23}{1680}\right) \frac{f^{\prime \prime 2}}{f^{2}}+\left(\frac{1}{32} \xi^{2}-\frac{11}{96} \xi+\frac{7}{320}\right) \frac{f^{\prime 4}}{f^{4}}
\end{aligned}
$$




$$
\begin{aligned}
& -\left(\frac{1}{2} \xi^{2}-\frac{1}{6} \xi+\frac{1}{72}\right) \frac{f^{\prime \prime}}{f r^{2}}+\left(\frac{1}{4} \xi^{2}-\frac{1}{12} \xi+\frac{1}{144}\right) \frac{f^{\prime 2}}{f^{2} r^{2}} \\
& +\frac{5 \xi-1}{30} \frac{f^{\prime \prime \prime} r^{\prime}}{f r}+\left(\frac{1}{2} \xi^{2}-\frac{13}{24} \xi+\frac{73}{560}\right) \frac{f^{\prime \prime} f^{\prime} r^{\prime}}{f^{2} r}+\left(\xi^{2}-\frac{1}{24} \xi-\frac{13}{1680}\right) \frac{f^{\prime \prime} r^{\prime \prime}}{f r} \\
& +\left(\frac{1}{2} \xi^{2}-\frac{1}{6} \xi-\frac{127}{2520}\right) \frac{f^{\prime \prime} r^{\prime 2}}{f r^{2}}-\left(\frac{1}{4} \xi^{2}-\frac{7}{24} \xi+\frac{23}{480}\right) \frac{f^{\prime 3} r^{\prime}}{f^{3} r}-\left(\frac{1}{2} \xi^{2}+\frac{1}{48} \xi-\frac{11}{480}\right) \frac{f^{\prime 2} r^{\prime \prime}}{f^{2} r} \\
& +\left(\frac{1}{4} \xi^{2}-\frac{1}{8} \xi-\frac{83}{2016}\right) \frac{f^{\prime 2} r^{\prime 2}}{f^{2} r^{2}}+\left(\frac{1}{6} \xi-\frac{11}{480}\right) \frac{f^{\prime} r^{\prime \prime \prime}}{f r}+\left(2 \xi^{2}-\frac{1}{2} \xi-\frac{401}{10080}\right) \frac{f^{\prime} r^{\prime \prime} r^{\prime}}{f r^{2}} \\
& +\left(\xi^{2}-\frac{5}{12} \xi+\frac{179}{840}\right) \frac{f^{\prime} r^{\prime 3}}{f r^{3}}-\left(\xi^{2}-\frac{5}{12} \xi+\frac{1}{24}\right) \frac{f^{\prime} r^{\prime}}{f r}+\left(\frac{1}{6} \xi-\frac{1}{80}\right) \frac{r^{\prime \prime \prime \prime}}{r} \\
& +\frac{5 \xi-1}{30} \frac{r^{\prime \prime \prime} r^{\prime}}{r^{2}}+\left(2 \xi^{2}-\frac{11}{12} \xi+\frac{163}{840}\right) \frac{r^{\prime \prime} r^{\prime 2}}{r^{3}}+\left(2 \xi^{2}-\frac{1}{6} \xi-\frac{97}{3360}\right) \frac{r^{\prime \prime 2}}{r^{2}} \\
& +\left(\frac{1}{2} \xi^{2}-\frac{61}{420}\right) \frac{r^{\prime 4}}{r^{4}}-\left(2 \xi^{2}-\frac{5}{6} \xi+\frac{7}{96}\right) \frac{r^{\prime \prime}}{r^{3}}-\left(\xi^{2}-\frac{1}{6} \xi\right) \frac{r^{\prime 2}}{r^{4}} \\
& +\left(\frac{1}{2} \xi^{2}-\frac{1}{6} \xi+\frac{1}{60}\right) \frac{1}{r^{4}} .
\end{aligned}
$$

The expression for $\left\langle\phi^{2}\right\rangle$, given by Eq. (28), has same order as the term proportionate to $m^{-2}$ in DeWitt-Schwinger expansion for $\left\langle\phi^{2}\right\rangle$, however it does not coincide with them. The reason of this is that the topological structures of Minkowski and wormhole spacetimes are different.

As a simple example, we consider the wormhole spacetime with the metric

$$
d s^{2}=-d t^{2}+d \rho^{2}+\left(\rho^{2}+r_{0}^{2}\right)\left(d \theta^{2}+\sin ^{2} \theta d \varphi^{2}\right),
$$

given by Morris and Thorne [5]. Here $f(\rho) \equiv 1$ and $r(\rho)=\left(\rho^{2}+r_{0}^{2}\right)^{1 / 2}$. The characteristic scale of varying of the metric functions can be defined as follows: $L^{-2} \sim r^{\prime \prime} / r=r_{0}^{2}\left(\rho^{2}+r_{0}^{2}\right)^{-2}$ near the throat, and $L^{-1} \sim r^{\prime} / r=\rho\left(\rho^{2}+r_{0}^{2}\right)^{-1}$ far from the throat. Stress that in both cases $r(\rho) \sim L$. Note also that the basic WKB condition $L / L_{\star} \gg 1$ is satisfied provided $r_{0} / r_{\mathrm{c}} \gg 1$, where $r_{\mathrm{c}}=m^{-1}$ is the Compton length. Carrying out appropriate calculations in Eq. (28) we find

$$
4 \pi^{2} m^{2}\left\langle\phi^{2}\right\rangle_{\mathrm{ren}}=\frac{\Psi_{\xi}(x)}{r_{0}^{4}}
$$

where

$$
\Psi_{\xi}(x)=-\frac{1}{\left(x^{2}+1\right)^{4}}\left(\frac{9}{70} x^{4}-\frac{117}{1120} x^{2}-\frac{5}{4} \xi x^{2}-\frac{1}{2} \xi^{2}+\frac{1}{21}\right)
$$

and $x=\rho / r_{0}$ is a dimensionless proper radial distance. The obtained formula obviously shows that the qualitative behavior of $\left\langle\phi^{2}\right\rangle$ is determined by the function $\Psi_{\xi}(x)$ and depends only on $\xi$. This dependance is very interesting. In Figs. 1,2 we present the family of curves of $\left\langle\phi^{2}\right\rangle$ for various values of $\xi$. It is seen that $\left\langle\phi^{2}\right\rangle$ is purely negative at $\xi=0$, and becomes alternating for greater values of $|\xi|$. To characterize this feature more accurate we consider an averaged value of $\left\langle\phi^{2}\right\rangle$, i.e.,

$$
\int_{-\infty}^{\infty}\left\langle\phi^{2}\right\rangle r(\rho) d \rho=\frac{1}{4 \pi^{2} m^{2} r_{0}^{2}} \int_{-\infty}^{\infty} \Psi_{\xi}(x) \sqrt{x^{2}+1} d x=\frac{1}{4 \pi^{2} m^{2} r_{0}^{2}}\left(\frac{8}{15} \xi^{2}+\frac{1}{3} \xi-\frac{937}{12600}\right) .
$$

It is easily to find that the averaged value is negative provided $\xi_{1}<\xi<\xi_{2}$, where $\xi_{1} \approx-0.7994$ and $\xi_{2} \approx 0.1744$, and positive in the other cases. At last, we note that the average value of $\left\langle\phi^{2}\right\rangle$, computed in the metric (29), is negative for $\xi=0$ (minimal coupling) and $\xi=1 / 6$ (conformal coupling).

\section{B. The case $r \ll L$}

Now let us analyse the analytical approximation (27) in the region $r(\rho) \ll L$. Remember that $L$ is the characteristic scale of varying of the metric, and so $L^{-1}$ is proportional to derivatives of the metric functions. Hence it follows from 
the relation $r(\rho) \ll L$ that one may neglect terms with derivatives in Eq. (27) with respect to terms without ones. As the result, we obtain

$$
4 \pi^{2}\left\langle\phi^{2}\right\rangle_{\text {ren }}=\frac{1}{4}\left[m^{2}+\frac{2}{r^{2}}\left(\xi-\frac{1}{6}\right)\right] \ln \left(\frac{\mu^{2}}{m_{\mathrm{DS}}^{2} r^{2}}\right)-\frac{\delta}{2 r^{2}}-\frac{\mu^{2}}{r^{2}} S_{0}(2 \pi \mu)
$$

It is worth to emphasize that an extreme example of the metric, for which the relation $r(\rho) \ll L$ is satisfied, is that when $r(\rho) \equiv r_{0}=$ constant and $f(\rho) \equiv f_{0}=$ constant, i.e.,

$$
d s^{2}=-f_{0} d t^{2}+d \rho^{2}+r_{0}^{2}\left(d \theta^{2}+\sin ^{2} \theta d \varphi^{2}\right) .
$$

To illustrate a case of the mertic with non-constant coefficients obeying the condition $r(\rho) \ll L$ we may consider the following example: $f(\rho) \equiv f_{0}=$ constant and $r^{2}(\rho)=r_{0}^{2}\left[1+\alpha^{2} \ln \left(1+\rho^{2} / \rho_{0}^{2}\right)\right]$, where $\alpha$ and $\rho_{0}$ are some parameters; the relation $r(\rho) \ll L$ is satisfied provided $\alpha r_{0} / \rho_{0} \ll 1$.

Let, in addition to $r(\rho) \ll L$, the relation $r / r_{\mathrm{c}} \gg 1$ be satisfied. Stress that it assumes that $m \neq 0$ and so $m_{\mathrm{DS}}=m$. In this case $\mu \approx m r$ and we can simplify Eq. (33) by using the asymptotical form (A1) for $S_{0}(2 \pi \mu)$ and taking into account that $\ln \left(\mu^{2} /\left(m_{\mathrm{DS}} r\right)^{2}\right)=\ln \left(1+2 \delta /(m r)^{2}\right) \approx 2 \delta /(m r)^{2}-2 \delta^{2} /(m r)^{4}$ :

$$
4 \pi^{2} m^{2}\left\langle\phi^{2}\right\rangle_{\mathrm{ren}}=\frac{1}{2 r^{4}}\left(\xi^{2}-\frac{\xi}{3}+\frac{1}{30}\right)
$$

It is easily to see that the last expression is positive for all values $\xi$.

Now consider the opposite relation $m r=r / r_{\mathrm{c}} \ll 1$. Note that it permits in particular the massless case $m=0$. In this case $\mu \approx(2 \delta)^{1 / 2}$ the expression (33) for $\left\langle\phi^{2}\right\rangle$ can approximately be rewritten as

$$
4 \pi^{2}\left\langle\phi^{2}\right\rangle_{\text {ren }}=\frac{1}{2 r^{2}}\left(\xi-\frac{1}{6}\right) \ln \left(\frac{2 \delta}{m_{\mathrm{DS}}^{2} r^{2}}\right)-\frac{\delta}{2 r^{2}}\left[1+4 S_{0}(2 \pi \sqrt{2 \delta})\right] .
$$

In particular, for conformal coupling $\xi=1 / 6(\delta=1 / 24)$ we obtain $S_{0}\left(3^{-1 / 2} \pi\right) \approx-0.0596$ and

$$
4 \pi^{2}\left\langle\phi^{2}\right\rangle_{\text {ren }}=-\frac{0.0159}{r^{2}}
$$

Finally stress that the obtained expression (33) for $\left\langle\phi^{2}\right\rangle$ is not approximate but exact in the $R^{2} \times S^{2}$ spacetime with the metric (34).

\section{ACKNOWLEDGMENT}

This work was supported by the Russian Foundation for Basic Research grant No 99-02-17941.

\section{APPENDIX: ASYMPTOTICS FOR $S_{N}(2 \pi \mu)$ AND $N_{N}^{M}(\mu)$}

In this appendix we obtain asymptotics at large values of argument for the functions $S_{n}(2 \pi \mu)$ and $N_{n}^{m}(\mu)$. Consider the functions $S_{n}(2 \pi \mu)$ defined by Eqs. (A1). Making the substitution $y=2 \pi \mu x$ in Eqs. (A1) we find

$$
\begin{aligned}
& S_{0}(2 \pi \mu)=\lambda^{2} \int_{0}^{\infty} \frac{y \ln \left|1-\lambda^{2} y^{2}\right|}{e^{y}+1} d y \\
& S_{n}(2 \pi \mu)=\int_{0}^{\infty} d y \ln \left|1-\lambda^{2} y^{2}\right| \frac{d}{d y}\left(\frac{1}{y} \frac{d}{d y}\right)^{n-1} \frac{y^{2(n-1)}}{e^{y}+1}
\end{aligned}
$$

where $\lambda=(2 \pi \mu)^{-1}$ and $\mu=\left(m^{2} r^{2}+2 \delta\right)^{1 / 2}$. Note that the integrands in Eqs. (A1) contain exponential functions and are exponentially decreasing at large values of $y$. Hence the main contribution into the integrals is provided with values of integrands in the region $0<y<1$. We are interesting in the case $\mu \gg 1$. In this case $\lambda \ll 1$ and $\lambda y \ll 1$ if $0<y<1$. Now we may use the asymptotical formula $\ln \left(1-\lambda^{2} y^{2}\right)=-\lambda^{2} y^{2}-\frac{1}{2} \lambda^{4} y^{4}-\frac{1}{3} \lambda^{6} y^{6}+\mathrm{O}\left(\lambda^{8} y^{8}\right)$. Substituting this into Eqs. (A1) and integrating gives 


$$
\begin{aligned}
& S_{0}(2 \pi \mu)=-\frac{7}{1920(m r)^{4}}+\mathrm{O}\left((m r)^{-6}\right), \quad S_{1}(2 \pi \mu)=\frac{1}{24(m r)^{2}}+\mathrm{O}\left((m r)^{-4}\right), \\
& S_{2}(2 \pi \mu)=\mathrm{O}\left((m r)^{-4}\right), \quad S_{3}(2 \pi \mu)=\mathrm{O}\left((m r)^{-6}\right) .
\end{aligned}
$$

Consider now the functions $N_{n}^{m}(\mu)$ :

$$
N_{n}^{m}(\mu)=\sum_{l=0}^{\infty} \frac{\left(l+\frac{1}{2}\right)^{m}}{\left[\mu^{2}+\left(l+\frac{1}{2}\right)^{2}\right]^{n}},
$$

where $n=2,3,4, \ldots$ and $m=1,3, \ldots, 2 n-3$. It is easily to check that they obey the following recurrent formula:

$$
N_{n}^{m}(\mu)=N_{n-1}^{m-2}(\mu)-\mu^{2} N_{n}^{m-2}(\mu),
$$

and in addition,

$$
N_{n}^{1}(\mu)=\frac{(-1)^{n+1}}{2^{n}(n+1) !}\left(\frac{1}{\mu} \frac{d}{d \mu}\right)^{n} N_{2}^{1}(\mu) .
$$

Using these formulas, one can express any function $N_{n}^{m}(\mu)$ via $N_{2}^{1}(\mu)$. The function $N_{2}^{1}(\mu)$ can be represented as follows [see Ref. [27], Eq. (5.1.26.23)]:

$$
N_{2}^{1}(\mu)=-\frac{i}{4 \mu}\left[\psi^{\prime}\left(\frac{1}{2}-i \mu\right)-\psi^{\prime}\left(\frac{1}{2}+i \mu\right)\right],
$$

where $\psi(z)=\Gamma^{\prime}(z) / \Gamma(z)$ is the digamma function. Using the asymptotical properties of the digamma function (see, for example, Ref. [28]) and taking into account Eqs. (A4), (A5) we can obtain the following asymptotic for large values of $\mu$ :

$$
\begin{aligned}
& N_{2}^{1}(\mu)=\frac{1}{2(m r)^{2}}-\frac{\xi-1 / 6}{(m r)^{4}}+\mathrm{O}\left((m r)^{-6}\right), \quad N_{3}^{1}(\mu)=\frac{1}{4(m r)^{4}}-\frac{\xi-1 / 6}{(m r)^{6}}+\mathrm{O}\left((m r)^{-8}\right), \\
& N_{3}^{3}(\mu)=\frac{1}{4(m r)^{2}}-\frac{\delta}{2(m r)^{4}}+\mathrm{O}\left((m r)^{-6}\right), \quad N_{4}^{1}(\mu)=\frac{1}{6(m r)^{6}}+\mathrm{O}\left((m r)^{-8}\right), \\
& N_{4}^{3}(\mu)=\frac{1}{12(m r)^{4}}+\mathrm{O}\left((m r)^{-6}\right), \quad N_{4}^{5}(\mu)=\frac{1}{6(m r)^{2}}+\mathrm{O}\left((m r)^{-4}\right), \\
& N_{5}^{1}(\mu)=\frac{1}{8(m r)^{8}}+\mathrm{O}\left((m r)^{-10}\right), \quad N_{5}^{3}(\mu)=\frac{1}{24(m r)^{6}}+\mathrm{O}\left((m r)^{-8}\right), \\
& N_{5}^{5}(\mu)=\frac{1}{24(m r)^{4}}+\mathrm{O}\left(\left(m r^{-6}\right), \quad N_{5}^{7}(\mu)=\frac{1}{8(m r)^{2}}+\mathrm{O}\left((m r)^{-4}\right),\right. \\
& N_{6}^{1}(\mu)=\frac{1}{10(m r)^{10}}+\mathrm{O}\left((m r)^{-12}\right), \quad N_{6}^{3}(\mu)=\frac{1}{40(m r)^{8}}+\mathrm{O}\left((m r)^{-10}\right), \\
& N_{6}^{5}(\mu)=\frac{1}{60(m r)^{6}}+\mathrm{O}\left((m r)^{-8}\right), \quad N_{6}^{7}(\mu)=\frac{1}{40(m r)^{4}}+\mathrm{O}\left((m r)^{-6}\right), \\
& N_{6}^{9}(\mu)=\frac{1}{10(m r)^{2}}+\mathrm{O}\left((m r)^{-4}\right) .
\end{aligned}
$$

[1] Of course, this 'definition' is not rigorous. For more details, see Ref. 22]. More recently Hochberg and Visser [23] gave a precise, local definition of a (traversable) wormhole throat and performed a detailed, pure local analysis of wormhole geometry. Also a fresh discussion devoted to a notion of a wormhole can be found in Ref. 24]

[2] L. Flamm, Phys. Z., 17, 48 (1916).

[3] A. Einstein and N. Rosen, Phys. Rev. 48, 73 (1935).

[4] J.A. Wheeler, Phys. Rev. 97, 511 (1955). 
[5] M. S. Morris and K. S. Thorne, Am. J. Phys. 56, 395 (1988).

[6] M. S. Morris, K. S. Thorne and U. Yurtsever, Phys. Rev. D 61, 1446 (1988).

[7] The reader can find some references on wormhole physics in the excelent book by Visser 22]

[8] A. Agnese and M. La Camera, Phys. Rev. D 51, 2011 (1995)

[9] K. K. Nandi, A. Islam, and J. Evans, Phys. Rev. D 55, 2497 (1997)

[10] L. A. Anchordoqui, S. Perez Bergliaffa, and D. F. Torres, Phys. Rev. D 55, 5226 (1997)

[11] D. Hochberg, Phys. Lett. B251, 349 (1990)

[12] B. Bhawal and S. Kar, Phys. Rev. D 46, 2464 (1992)

[13] C. Barceló and M. Visser, Phys. Lett. B466, 127 (1999)

[14] C. Barceló and M. Visser, gr-qc/0003025

[15] S. V. Sushkov, Phys. Lett. A164, 33 (1992)

[16] V. P. Frolov and A. I. Zel'nikov, Phys. Rev. D 35, 3031 (1987).

[17] D. Hochberg, A. Popov, and S. V. Sushkov, Phys. Rev. Lett. 78, 2050 (1997).

[18] P. R. Anderson, W. A. Hiscock, and D. A. Samuel, Phys. Rev. D 51, 4337 (1995).

[19] B. E. Taylor, W. A. Hiscock, and P. R. Anderson, Phys. Rev. D 55, 6116 (1997).

[20] V. Khatsymovsky, Phys. Lett. B429, 254 (1998)

[21] S. V. Sushkov, gr-qc/0001058, to appear in Phys. Rev. D

[22] M. Visser, Lorentzian Wormholes: From Einstein to Hawking (American Institute of Physics, New York, 1995).

[23] D. Hochberg and M. Visser, Phys. Rev. D 56, 4745 (1997)

[24] S. Krasnikov, gr-qc/9909016, to appear in Phys. Rev. D

[25] P. Candelas and K. W. Howard, Phys. Rev. D 29, 1618 (1984).

[26] S. M. Christensen, Phys. Rev. D 14, 2490 (1976).

[27] A.P. Prudnikov, Yu.A. Brychkov, and O.I. Marichev, Integrals and Series, vol. 1, (Gordon and Breach, Amsterdam, 1996).

[28] M. Abramowitz and I.A. Stegun, Handbook of Mathematical Functions, (US National Bureau of Standards, Washington, 1964). 


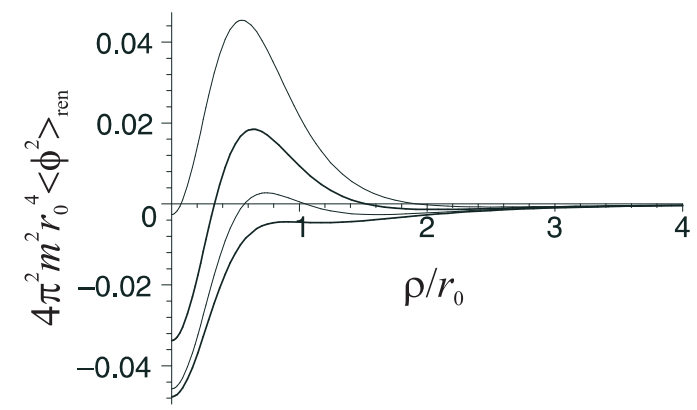

FIG. 1. The curves in this figure display the the WKB approximation for $\left\langle\phi^{2}\right\rangle$ (28) calculated in the large $m$ limit in the Morris-Thorne metric (29). From bottom to top the curves correspond to the values $\xi=0,1 / 16,1 / 6,3 / 10$.

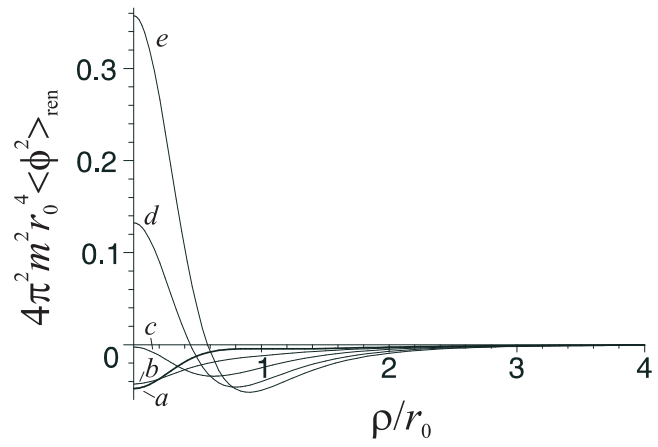

FIG. 2. The curves in this figure display $\left\langle\phi^{2}\right\rangle$ calculated for negative values of $\xi$. The labels $a, b, c, d, e$ mark curves which correspond to the values $\xi=0,-0.1,-0.3,-0.6,-0.9$. 